\title{
The Decompensated Cirrhosis Care Bundle for Acute Admissions: Implementation and Impact
}

\author{
Cargill Z, Tang K and Alisa A* \\ Department of Gastroenterology, Barnet Hospital, UK
}

Submission: March 03, 2017; Published: March 27, 2017

"Corresponding author: Alisa A, Department of Gastroenterology, Barnet Hospital, Royal Free NHS Foundation Trust, UK, Email: akeelalisa@yahoo.com

\section{Introduction}

Acute Decompensated Cirrhosis (ADC) is a medical emergency that carries high mortality (10-20\%). The British Society of Gastroenterology/British Association for the Study of the Liver has published an evidenced based Decompensated Cirrhosis Care Bundle (DCCB) checklist to be completed during the initial six hours of admission for all patients with ADC and for expert care to be implemented within 24 hours.

\section{Methods}

Patients were identified by the gastroenterology team inpatient referral system, admission notes and electronic records. It included 22 consecutive admissions with cirrhosis between18th April 2016 and 18th June 2016. This was following introduction of a modified liver care bundle into the trust. The aim was to compare our performance and outcomes after the introduction of the preformed to data collected prior to implementation.

\section{Results}

Twenty-two admissions with ADC were identified over the twomonth period in April to June 2016. Performance prior to and post DCCB introduction can be seen in table 1 . There was a substantial improvement in the numbers of imaging requested and early ascitic taps. Additionally, post DCCB implementation, a greater number of admissions with acute kidney injury or hyponatremia had fluid resuscitation with the recommended fluid regimen. However, the management of those with a suspected upper gastrointestinal bleed differed little. Inpatient mortality during data collection was $0 \%$ compared to $18 \%$ pre DCCB introduction which? Related to excess winter mortality/sicker patients at the time/?? (Table 1)

Table 1

\begin{tabular}{|c|c|c|}
\hline & $\begin{array}{c}\text { Pre DCCB } \\
\text { Dec 2016- Feb } 2016\end{array}$ & $\begin{array}{l}\text { DCCB Implementation } \\
\text { Apr } 2016 \text { - Jun } 2016\end{array}$ \\
\hline & \multicolumn{2}{|c|}{ Demographics } \\
\hline $\mathrm{N}$ & 40 & 22 \\
\hline Average length of stay & -x 10 days [range $3-42$ days] & 13 days(range $2-57$ ) \\
\hline Sex Male & $20(50 \%)$ & $11(50 \%)$ \\
\hline Sex Female & $20(50 \%)$ & $11(50 \%)$ \\
\hline Age & $-\mathrm{x}=57$ years(range $33-86$ years) & $-x=56$ years(range $37-76$ years) \\
\hline \multicolumn{3}{|c|}{ Aetiology of Cirrhosis } \\
\hline $\mathrm{N}$ & 40 & 22 \\
\hline Alcoholic Liver Disease & $29(73 \%)$ & $17(77 \%)$ \\
\hline NASH & $3(8 \%)$ & $3(14 \%)$ \\
\hline Cryptogenic & $4(10 \%)$ & $0(0 \%)$ \\
\hline $\mathrm{HCV}$ & $2(5 \%)$ & $0(0 \%)$ \\
\hline Hemochromatosis & $1(3 \%)$ & $0(0 \%)$ \\
\hline
\end{tabular}




\section{Advanced Research in Gastroenterology \& Hepatology}

\begin{tabular}{|c|c|c|}
\hline HBV & $0(0 \%)$ & $1(5 \%)$ \\
\hline Unknown & $1(3 \%)$ & $2(10 \%)$ \\
\hline \multicolumn{3}{|c|}{ Early Investigations } \\
\hline $\mathrm{N}$ & 40 & 22 \\
\hline New Early Warning Scores & $40(100 \%)$ & $22(100 \%)$ \\
\hline FBC, U\&E, CRP & $40(100 \%)$ & $22(100 \%)$ \\
\hline Bone profile and Magnesium & $25(63 \%)$ & $14(64 \%)$ \\
\hline Coagulation profile & $37(85 \%)$ & $22(100 \%)$ \\
\hline Ascitic tap if clinical ascites present & $11 / 29(38 \%)$ & $9(75 \%)$ \\
\hline Ultrasound Abdomen/Imaging & $24(60 \%)$ & $18(82 \%)$ \\
\hline \multicolumn{3}{|c|}{ Recent Excessive Alcohol Consumption } \\
\hline $\mathrm{N}$ & 25 & 9 \\
\hline IV Pabrinex & $25(100 \%)$ & $9(100 \%)$ \\
\hline CIWA score +alcohol reducing regimen & $24(96 \%)$ & $9(100 \%)$ \\
\hline \multicolumn{3}{|c|}{ Suspected Infection } \\
\hline $\mathrm{N}$ & 19 & 8 \\
\hline Started on Antibiotics & $19(100 \%)$ & $8(100 \%)$ \\
\hline Blood cultures & $7(37 \%)$ & $3(38 \%)$ \\
\hline \multicolumn{3}{|c|}{ Acute Kidney Injury and/or Hyponatraemia } \\
\hline $\mathrm{N}$ & 19 & 6 \\
\hline Fluid resuscitation with sodium chloride & $18(86 \%)$ & $6(100 \%)$ \\
\hline Fluid balance chart & $20(95 \%)$ & $6(100 \%)$ \\
\hline Weight chart & $4(19 \%)$ & $1(17 \%)$ \\
\hline \multicolumn{3}{|c|}{ Suspected GI Bleeding } \\
\hline $\mathrm{N}$ & 14 & 5 \\
\hline Endoscopy within 24 hours & $13(93 \%)$ & $4(80 \%)$ \\
\hline Terlipressin administration & $10(71 \%)$ & $3(60 \%)$ \\
\hline Antibiotics & $11(79 \%)$ & $1(20 \%)$ \\
\hline Red Blood Cells transfusion & $11(79 \%)$ & $4(80 \%)$ \\
\hline FFP transfusion & $3(21 \%)$ & $0(0 \%)$ \\
\hline \multicolumn{3}{|c|}{ Encephalopathy } \\
\hline $\mathrm{N}$ & 40 & 22 \\
\hline Signs of encephalopathy & $23(58 \%)$ & $5(23 \%)$ \\
\hline \multicolumn{3}{|c|}{ Gastroenterology Review } \\
\hline $\mathrm{N}$ & 40 & 22 \\
\hline Within 24 hours & $33(83 \%)$ & $21(95 \%)$ \\
\hline
\end{tabular}

\section{Discussion and Conclusion}

Introduction and implementation of the Decompensated Care Bundle is at its early stages. Already we have seen improvements in prompt management of ascites and timely arrangement of imaging.
We would expect progress to continue as staff become more familiarised with the DCCB. To ensure effective use across our trust, accessibility and further education of staff should be addressed. Ongoing audit and assessment will help assess patient outcomes. 


\section{Your next submission with Juniper Publishers will reach you the below assets}

- Quality Editorial service

- Swift Peer Review

- Reprints availability

- E-prints Service

- Manuscript Podcast for convenient understanding

- Global attainment for your research

- Manuscript accessibility in different formats

( Pdf, E-pub, Full Text, Audio)

- Unceasing customer service

Track the below URL for one-step submission https://juniperpublishers.com/online-submission.php 